\title{
Der BREXIT und andere „Unfälle“
}

\section{Tiefere Ursachen und Konsequenzen für die deutsche Wirtschaft}

\author{
Max Otte (iD
}

Online publiziert: 18. April 2017

(C) Der/die Autor(en) 2017. Dieser Artikel ist eine Open-Access-Publikation.

Zusammenfassung Am 23. Juni 2016 geschah das Unerwartete: Die britische Bevölkerung stimmte mehrheitlich für einen Austritt Großbritanniens aus der europäischen Union. Premierminister David Cameron, der sich häufig kritisch über die Europäische Union geäußert hatte, dieses Ergebnis aber bestimmt nicht wollte, bekam die Quittung für seine Politik.

Das BREXIT-Referendum, wie auch die Wahl Donald Trumps zum 45. Präsidenten der USA sowie das gescheiterte Verfassungsreferendum in Italien sind dabei Symptome einer tiefgreifenden Krise der globalen Ordnung. Diese Krise ist real. Sie löst Unsicherheit bei den Funktionseliten aus. Aber sie ist auch - um ein viel benutztes Klischee noch weiter zu strapazieren - eine Chance.

In meinen Ausführungen werde ich die Ursachen und Manifestationen der tiefgreifenden Krise am Beispiel des BREXIT beleuchten. Ich werde zweitens argumentieren, dass Protektionismus nicht immer schlecht sein muss, sondern dass es sich, wie bei eigentlich allen politischen Entscheidungen, um eine Frage von Maß und Mitte handelt. Und ich werde drittens skizzieren, wie sich die deutsche Wirtschaft und Industrie in einem veränderten Umfeld positionieren sollen - denn neben Großbritannien hat eine ähnliche Entwicklung in den USA bereits eingesetzt.

Dabei werde ich mich der etwas aus der Mode gekommenen politischen Ökonomie bedienen und berücksichtigen, dass eine Wirtschaftsordnung immer auch einen Interessausgleich zwischen Interessengruppen und Klassen leisten muss, wenn sie auf Dauer legitim sein soll. Zudem muss auch der Ausgleich zwischen Ländern und Regionen gelingen. Damit befinde ich mich in der besten Tradition des von mir

Max Otte, Ph. D. (Princeton University) ist Gründer und Direktor des IFVE Institut für Vermögensentwicklung in Köln. Von 2011 bis 2016 war er Universitätsprofessor an der Karl-Franzens-Universität in Graz. Von seiner Professur (C-3) an der Hochschule Worms ist er beurlaubt.

M. Otte $(\bowtie)$

IFVE Institut für Vermögensentwicklung GmbH, Köln, Deutschland

E-Mail: max@otte.org 
verehrten Friedrich Lists, aber auch Francois Quesnays, John Maynard Keynes', Karl Marx' und meines Lehrers Robert Gilpin. Wer, wie die meisten zeitgenössischen Ökonomen, die aktuellen politischen Phänomene ausschließlich auf individuelle Konsum- und Produktionsentscheidungen zurückführen will, wird ihnen nicht gerecht. Und er wird sie nicht verstehen.

Der Dekan der Wirtschaftswissenschaften der Universität Graz äußerte auf einer Veranstaltung mit heterodoxen Ökonomen und dem Verfasser im Jahr 2012, dass die Wirtschaftswissenschaften mittlerweile sehr gut darin seien, zu analysieren, wie sich Regeln auswirken. Es gäbe aber große Lücken bei den Ökonomen, zu verstehen, wie Regeln gemacht werden. Genau das ist die Aufgabe der politischen Ökonomie. Wer bestimmt die Regeln in welchem Fall, und wem nützt es? Dies ist die Kernfrage, und sie wird viel zu selten gestellt. In diesem Beitrag beleuchte ich, wie der BREXIT und verwandte Ereignisse die globalen Regeln beeinflussen könnten.

I sympathize, therefore, with those who would minimize, rather than with those who would maximize, economic entanglement among nations. Ideas, knowledge, science, hospitality, travel - these are the things which should of their nature be international. But let goods be homespun whenever it is reasonably and conveniently possible, and, above all, let finance be primarily national. (...)

John Maynard Keynes (1933)

Am 23. Juni 2016 geschah das Unerwartete: Die britische Bevölkerung stimmte mehrheitlich für einen Austritt Großbritanniens aus der europäischen Union. Premierminister David Cameron, der sich häufig kritisch über die Europäische Union geäußert hatte, dieses Ergebnis aber bestimmt nicht wollte, bekam die Quittung für seine Politik.

Das BREXIT-Referendum, wie auch die Wahl Donald Trumps zum 45. Präsidenten der USA sowie das gescheiterte Verfassungsreferendum in Italien sind dabei Symptome einer tiefgreifenden Krise der globalen Ordnung. Diese Krise ist real. Sie löst Unsicherheit bei den Funktionseliten aus. Aber sie ist auch - um ein viel benutztes Klischee noch weiter zu strapazieren - eine Chance.

In meinen Ausführungen werde ich die Ursachen und Manifestationen der tiefgreifenden Krise am Beispiel des BREXIT beleuchten. Ich werde zweitens argumentieren, dass Protektionismus nicht immer schlecht sein muss, sondern dass es sich, wie bei eigentlich allen politischen Entscheidungen, um eine Frage von Maß und Mitte handelt. Und ich werde drittens skizzieren, wie sich die deutsche Wirtschaft und Industrie in einem veränderten Umfeld positionieren sollen - denn neben Großbritannien hat eine ähnliche Entwicklung in den USA bereits eingesetzt.

Dabei werde ich mich der etwas aus der Mode gekommenen politischen Ökonomie bedienen und berücksichtigen, dass eine Wirtschaftsordnung immer auch einen Interessausgleich zwischen Interessengruppen und Klassen leisten muss, wenn sie auf Dauer legitim sein soll. Zudem muss auch der Ausgleich zwischen Ländern und Regionen gelingen. Damit befinde ich mich in der besten Tradition des von mir verehrten Friedrich List, aber auch von Francois Quesnay, John Maynard Keynes, Karl Marx und meines Lehrers Robert Gilpin. Wer, wie die meisten zeitgenössischen Ökonomen, die aktuellen politischen Phänomene ausschließlich auf individu- 
elle Konsum- und Produktionsentscheidungen zurückführen will, wird ihnen nicht gerecht. Und er wird sie nicht verstehen.

Der Dekan der Wirtschaftswissenschaften der Universität Graz äußerte auf einer Veranstaltung mit heterodoxen Ökonomen und dem Verfasser im Jahr 2012, dass die Wirtschaftswissenschaften mittlerweile sehr gut darin seien, zu analysieren, wie sich Regeln auswirken (Scheuer et al. 2012). Es gebe aber große Lücken bei den Ökonomen, zu verstehen, wie Regeln gemacht werden. Genau das ist die Aufgabe der politischen Ökonomie. Wer bestimmt die Regeln in welchem Fall, und wem nützt es? Dies ist die Kernfrage, und sie wird viel zu selten gestellt. In diesem Beitrag beleuchte ich, wie der BREXIT und verwandte Ereignisse die globalen Regeln beeinflussen könnten.

The decadent international but individualistic capitalism, in the hands of which we found ourselves after the war, is not a success. It is not intelligent, it is not beautiful, it is not just, it is not virtuous - and it doesn't deliver the goods. In short, we dislike it, and we are beginning to despise it. But when we wonder what to put in its place, we are extremely perplexed.

John Maynard Keynes (1933, S. 758)

\section{Der BREXIT als Symptom einer tiefgreifenden Krise der ,liberalen Wirtschaftsordnung“}

Die ,vier Freiheiten“ - freie Personen, Waren, Dienstleistungs- und Kapitalverkehr - stellen neben der gemeinsamen Währung die Basis der europäischen Wirtschaftsund Währungsunion dar. Bereits 1998 prognostizierte ich, dass die Imbalance zwischen Waren, Dienstleistungs- und Kapitalverkehr auf der einen und (faktischer) Mobilität der Arbeitskräfte auf der anderen Seite zu einer tiefgreifenden demokratischen Legitimationskrise der Institutionen der Europäischen Union und zu einem Kollaps des ,alten Regimes“ („Regime“ als politikwissenschaftlich-systemtheoretischer Begriff) von Brüssel führen würde (Otte 1998). Genau dies passierte, als sich die Kapitalströme in Richtung Südeuropa mit Einsetzen der sogenannten Eurokrise umkehrten. Meine zweite Prognose, dass wir dann ein bürgernäheres und demokratisch besser legitimiertes Europa bekommen würden, harrt allerdings noch auf ihr Eintreffen. Im Gegenteil: Es sieht danach aus, dass unser Wirtschaftssystem immer unfreier wird.

„Freihandel“ ist spätestens seit Unterzeichnung des Allgemeinen Zoll- und Handelsabkommens GATT im Jahr 1947 eine der Grundsäulen des Weltwirtschaftssystems geworden, genauso wie Freihandel die offizielle Doktrin für die Welt zwischen 1846 und 1914 war. Die Basis der Theorie der komparativen Kostenvorteile wurde von David Ricardo in seinen „Principles of Political Economy and Taxation“ entwickelt (Ricardo 1815, 1817). Es macht für Länder auch dann Sinn, Handel zu treiben, wenn ein Land in jedem einzelnen Waren- und Dienstleistungssektor absolute Kostenvorteile besitzt. Durch Handel würde es allen Ländern besser gehen, und jedes Land würde sich auf die Güter und Dienstleistungen spezialisieren, bei denen es relative Vorteile besitzt. 
Dabei muss unbedingt berücksichtigt werden, dass die Debatte um Freihandel in Großbritannien vor einem sehr realen politischen Hintergrund mit realen politischen Interessen erfolgte. Das ist auch bei der heutigen Diskussion angebracht. In der ersten Hälfte des 19. Jahrhunderts hatten in England die adligen Großgrundbesitzer im Parlament Gesetze durchgebracht, die ab 1815 den Import von Getreide (Weizen, Hafer, Roggen) mit hohen Zöllen belegten. In Preisen von 2015 wurden damit ungefähr 200 Pfund je $13 \mathrm{~kg}$ Weizen (,quarter hundredweight“) gezahlt (Wikipedia 2017). Dies schützte die Einkommen des Adels und benachteiligte sowohl Produzenten als auch Industriearbeiter. Unter den Letzteren brachen regelmäßig Hungersnöte aus. Nach der großen Hungersnot in Irland in 1845 und 1846 bekamen die Befürworter eines „Repeal“ der Getreidegesetze die Oberhand. England hob die Gesetze zum Schutz der Getreideproduzenten auf. Auch als in den letzten Jahrzehnten des 19. Jahrhunderts die Konkurrenz vor allem durch billiges amerikanisches Getreide sehr groß wurde, behielt England anders als fast alle europäischen Länder seine Freihandelspolitik bei.

England war ab 1846 unbestreitbar der Champion des globalen Freihandels. Allerdings - und das ist eine der entscheidenden Voraussetzungen, die gerne von Ökonomen übersehen wird - konnte sich Großbritannien diese Freihandelspolitik nur deswegen erlauben, weil die Royal Navy zum dominanten Machtfaktor auf den Weltmeeren geworden und die Versorgung Großbritanniens mit Getreide nicht gefährdet war.

Charles Kindleberger legte 1973 in seinem Buch „The World in Depression“ die Basis für das, was später „Hegemonic Stability Theory“ genannt werden sollte: „For the world economy to be stable, there needs to be a stabilizer, one stabilizer" (Kindleberger 2013). Ein Hegemon, eine Führungsmacht müssten die Regeln (z. B. einer liberalen Weltwirtschaftsordnung) durchsetzen, von denen letztlich alle profitierten. In der Zeit nach 1846 war dies eindeutig England. Zwar würde der Hegemon spezielle Privilegien in Anspruch nehmen, aber die Stabilität und Berechenbarkeit der Wirtschaftsordnung würde letztlich allen nutzen. In der Zwischenkriegszeit 1919 bis 1939 konnte England diese Rolle nicht mehr erfüllen, und die USA wollten es nicht. In diese Zeit fielen die Große Depression sowie das Aufkommen autoritärer Regimes. Seit der Bretton-Woods-Konferenz im Jahr 1944 waren die USA der Champion einer liberalen Weltwirtschaftsordnung.

Nach 1989 bestand die Hoffnung, dass durch den Zusammenbruch des Kommunismus nun die liberale Weltwirtschaftsordnung auf die ganze Welt ausgedehnt werden könne. Das Buch „The End of History“ von Francis Fukuyama symbolisierte diese Hoffnung (Fukuyama 1992). Mit dem Vertrag von Maastricht zur Etablierung der europäischen Wirtschafts- und Währungsunion (1992), der Forcierung der Welthandelsorganisation WTO (1994), dem nordamerikanischen Freihandelsabkommen NAFTA (1994) sowie in jüngerer Zeit der Trans-Pacific Partnership und dem Transatlantischen Freihandelsabkommen (TTIP) schien es, als ob Fukuyamas Thesen sich erfüllten. Gleichzeitig setzen aber die USA, die Führungsmacht des Westens, zunehmend das Prinzip des Wirtschaftskriegs und der Sanktionen ein, zuletzt gegen Russland. „Wandel durch Handel“ wird zumindest teilweise wieder durch ökonomische Konfrontation ersetzt. Bereits 1971 hatten die USA durch den unilateralen 
Rückzug aus dem Weltwährungssytem von Bretton Woods einen ersten Schritt zur Aufgabe der alten Prinzipien gemacht.

Mein Lehrer Robert Gilpin hat in War and Change in World Politics und später in The Political Economy of International Relations auf Basis der Theorie der hegemonialen Stabilität eine Zyklentheorie entwickelt, welche in einem Vier-PhasenSchema die Fluktuationen im Grad der Offenheit und der Stabilität der internationalen Ordnung erklären soll (Gilpin 1983, 1987): 1. Durch geografische Vorteile, bessere Produktionsmethoden oder sonstige Ereignisse erlangt eine Nation eine dominante Stellung in der Weltwirtschaft. 2. Diese Nation (der „Hegemon“) nutzt seine dominante Position, um verbindliche Regeln in der Weltwirtschaft durchzusetzen, von denen er zwar am meisten, alle anderen aber ebenfalls profitieren. 3. Auf Dauer führen die Lasten, aber auch die Privilegien der hegemonialen Rolle zu einem Schwinden der Innovations- und Produktivkräfte. 4. Neue Wirtschaftsmächte entstehen und wollen die Regeln aktiv mitgestalten. 5. In der multi- oder bipolaren Phase des Übergangs von einem Hegemon auf den nächsten ist das Weltwirtschaftssystem instabil. In der Vergangenheit ist es dann regelmäßig zu großen Kriegen gekommen, so nach 1914, als die USA und Deutschland sich anschickten, England zu beerben, oder als England Spanien und Holland vom Zentrum der Weltbühne verdrängte.

Die USA stehen nicht mehr für $40 \%$ des Welt-BIP wie 1960, sondern nur noch für $20 \%$. Aktuell macht das das chinesische BIP $61 \%$ der U.S.-amerikanischen Wirtschaftsleistung aus. Gemessen an der Kaufkraftparität ist das chinesische BIP mittlerweile sogar größer als das amerikanische (Desjardins 2015). Das Weltwirtschaftssystem befindet sich wieder in einer multipolaren Phase des Übergangs (Patton 2016). BREXIT, „America first“, internationale Unruhen und populistische Bewegungen sind Symptome dieser Phase. Wir wissen noch nicht, wie die globale Wirtschaftsordnung der Zukunft aussehen wird. Mit großer Wahrscheinlichkeit wird sie sich jedoch von der aktuellen Ordnung unterscheiden. Und hoffentlich bleiben wir von größeren Kriegen, wie sie in solchen Phasen in der Vergangenheit regelmäBig aufgetreten sind, verschont.

\section{Die Zukunft: Protektionismus und Re-Nationalisierung?}

Letztlich waren es nicht primär wirtschaftliche Probleme, welche zum Erfolg des Brexit-Referendums führten, sondern die Sorgen um unkontrollierte Einwanderung und nationale Souveränität. Eine Mehrheit der Briten fühlte sich nicht mehr wohl mit einer Wirtschaftsordnung, in welcher viele das Land und die Menschen betreffende Entscheidungen zunehmend außerhalb des britischen Parlaments fielen. Diese ,populistischen“ Sorgen sind angesichts einer komplexen und zunehmend undurchschaubaren europäischen und globalen Wirtschaftsordnung und angesichts der Aushöhlung der Demokratie aus Sicht des Verfassers durchaus verständlich. Der Slogan „America First“ des amerikanischen Präsidenten Donald Trump zielt in eine ähnliche Richtung. Er wurde von den Medien weitgehend als eine „Kampfansage an die liberale Wirtschaftsordnung" gesehen (Hulverscheidt 2016; von Marschall 2016). 
Dabei darf nach Ansicht des Verfassers durchaus die Frage gestellt werden, ob die Liberalisierung nicht zu weit gegangen, ob das Auseinanderdriften von zunehmend irrelevanter politischer Willensbildung in den Parlamenten und der Art und Weise, wie durch Lobbyismus ökonomische Entscheidungen gefällt werden, nicht kontraproduktiv ist. Im 19. Jahrhundert stellte sich für viele Ökonomen die soziale Frage, die Frage, wie es sein konnte, dass es trotz ungeahnten Wirtschaftswachstums weiter Massenarmut und Elend gab. Der neue soziale Kluft des 21. Jahrhunderts besteht zwischen den großen Kapitalvermögen und der internationalen Funktionselite in Konzernen, Investmentgesellschaften, Medien, Politik und Stiftungen - den international mobilen Produktionsfaktoren auf der einen Seite - und Arbeitnehmern, kleinerem Mittelstand oder regional gebundenen Unternehmen - den immobilen Produktionsfaktoren auf der anderen Seite. Die Frage, ob die negativen Auswüchse des globalen Hyperkapitalismus nicht so groß geworden sind, dass es zu einer weiteren, größeren Krise kommen muss, wird zunehmend gestellt (Sinn 2017; Streek 2016; Wagenknecht 2016).

Internationale Kapitaltransaktionen sind in Sekundenbruchteilen möglich, Güterund vor allem Personenströme reagieren viel langsamer. Wenn diese Asymmetrie nicht durch eine entsprechende Markt- und Wirtschaftsordnung behoben wird, sind die international mobilen Akteure gegenüber den regional und national gebundenen im Vorteil. Die Letzteren werden dann durch „Standortwettbewerb“ erpressbar. Der Mehrwert wandert in Richtung der mobilen Faktoren. Dies passiert: Finanzströme brausen ungefesselt um den Globus und führen $\mathrm{zu}$ massiver Fehlallokation von Kapital sowie Boom-Bust-Zyklen, wie zum Beispiel in Asien nach 1997, in Irland nach der Finanzkrise 2008, in Südeuropa - verstärkt durch die Fehlkonstruktion Euro - nach 2010, mehrfach in Mexiko und anderswo. Der durch diese Instabilität angerichtete Schaden ist immens.

Die populistischen Forderungen (Brexit = Great Britain first, America first) können auch so gelesen werden, dass ein Land zuerst vor seiner eigenen Haustür kehren und die eigene Wirtschaft, Gesetzgebung und Gesellschaft in Ordnung bringen sollte. Wenn Irland ein laxes Steuerregime einführt und Konzerne einfach Gesellschaften dorthin verlagern, während die Produktion woanders bleibt, ist das dysfunktional und führt zu einer Benachteiligung der immobilen Faktoren. Die Wertschöpfung kommt dort, wo sie entstanden ist, nicht in Form von Steuern an.

Gestützt durch massive Lobbymacht scheinen die Regeln aber noch weiter zu Gunsten der Kapitalseite verändert zu werden. Auch bei TTIP geht es nicht prinzipiell um ,mehr Handel“ - die positiven Zusatzeffekte sind auch in den Augen der TTIP-Befürworter eher bescheiden ${ }^{1}$ - sondern darum, dass die Regeln noch günstiger für die Kapitalseite sind. Auch die politische und rechtsstaatliche Kontrolle wird zunehmend schwieriger. Wenn auf facebook in Deutschland ggf. strafrechtlich relevante Inhalte gepostet werden, das zuständige Gericht aber in Irland ist und alle Akten erst ins Englische übersetzt werden müssen, ist das schlichtweg inakzeptabel und ein Politikversagen ersten Ranges. Hinzu kommt die Tatsache, dass die sich überlappenden Jurisdiktionen (national, Europäische Union, Global) zunehmend un-

\footnotetext{
${ }^{1}$ So rechnen Erixon und Brauer mit einer dynamischen BIP-Steigerung von von 0,23 bzw. 0,47\% in der EU und 0,99 bis $1,33 \%$ in den USA (nach Freytag et al. 2014).
} 
überschaubarer und komplexer werden und auch die Planungssicherheit gerade für kleinere und mittlere Akteure verhindert wird. Fast fühlt man sich mit Thierry Baudet an das Mittelalter vor Einführung des Rechts- und Nationalstaats erinnert, in dem ebenfalls sich überlappende Jurisdiktionen von Kaiser und Papst um Einfluss rangen - zu Lasten von Wirtschaft und Menschen (Baudet 2012).

Das Auseinanderfallen von politischer (Ohn-)Macht und wirtschaftlicher Macht wurde von John Maynard Keynes schon 1933 kritisch gesehen: Das Auseinanderfallen von Eigentum und der wirklichen Verantwortung des Managements ist schon innerhalb eines Landes ein Problem, wenn bei Aktiengesellschaften zahllose Eigentümer heute kaufen und morgen verkaufen und dabei keinerlei Wissen über und Verantwortung gegenüber dem haben, was sie gerade besitzen (Keynes 1933, S. 756).

Noch schwieriger wird es laut Keynes, falls die internationale Dimension zu stark wird: „Wenn dasselbe Prinzip international angewendet wird, ist es in Zeiten der Belastungen unhaltbar und unerträglich - ich verhalte mich unverantwortlich gegenüber meinem Eigentum, und die, die es managen, sind unverantwortlich mir gegenüber. Ja, es mag eine Finanzkalkulation geben, die zeigt, dass es vorteilhaft ist, wenn meine Ersparnisse in den letzten Winkel der bewohnbaren Welt investiert werden, wo es den größten Grenznutzen des Kapitals oder die höchsten Zinsen gibt. Aber die Erfahrungen mehren sich, dass der Abstand zwischen Eigentum und Management ein Übel für die Beziehung zwischen den Menschen ist, der wahrscheinlich oder sogar sicherlich langfristig Spannungen produzieren wird, welche die reine Finanzkalkulation zunichte machen (Keynes 1933, S. 756). “

Aber nicht nur zwischen immobilen und mobilen Produktionsfaktoren müssen politische Regeln für einen vernünftigen Ausgleich sorgen. Auch bei der Handelsgesetzgebung und bei der Regulierung ist zwischen verschiedenen Ländern und Wirtschaftsblöcken entweder echte Supranationalität oder ansonsten „Waffenparität" geboten. Beides haben wir derzeit nicht. Insbesondere Europa scheint - mit einigen Ausnahmen in der Handelsgesetzgebung - hier zunehmend zum Objekt der Politik zu verkommen (Otte 2012).

Das hat Folgen: Nicht-kontinentaleuropäische Akteure drücken Kontinentaleuropa ihre Politik auf. Diese Asymmetrien in den Verhandlungspositionen haben die USA gegen über Europa in den letzten Jahren massiv genutzt: Mit der potenziellen Drohung, den Zugang zum größten Markt der Welt zu verschließen, wurden europäischen Konzernen die amerikanischen Regeln aufgezwungen - Siemens, Deutsche Bank und VW sind nur einige Beispiele. Die Schweizerische Bankenaufsicht agiert mittlerweile fast wie eine nachgeordnete Behörde des U.S. Department of Justice. Zudem sind die Belastungen hochgradig asymmetrisch: Während das U.S. Department of Justice von der Deutschen Bank für Subprime-Papiere, die noch nicht einmal an Privatanleger verkauft wurden, 16 Mrd. Dollar Buße forderte, kam Wells Fargo, die eine halbe Million Konten falsch angelegt hatte, mit der höchsten Strafe davon, die jemals an die amerikanische Verbraucherschutzbehörde erhoben hat: 185 Mio. Dollar (Korkery 2016). Der Gesamtschaden der VW-Affäre wird auf über $40 \mathrm{Mrd}$. Euro geschätzt (Jahberg 2015). In der GM-Affäre, in der defekte Zündschlösser 125 Menschen das Leben kosteten, betrug die Strafe eine Milliarde Dollar (Ivory und Vlasic 2015). Zudem wird Kontinentaleuropa (wo die Finanzkrise nicht ihren Ursprung hatte), mit raschen Schritten von einem bank- und kreditorientierten 
Wirtschaftssystem (das gut funktionierte) zu einem kapitalmarktbasierten System umgebaut (Otte 2010). Wenn allerdings eine Mannschaft, die American Football spielt, eine Fußballmannschaft zwingt, das Spiel nach ihren Regeln zu spielen, wird die Fußballmannschaft haushoch verlieren.

Es mag also durchaus sinnvoll sein, die nationale Kontrolle zumindest in Teilbereichen wieder zu stärken. Auch regionale Zusammenschlüsse wie die EU können effektiv sein, wenn sie richtig konstruiert sind. Es geht eben in der realen Handelspolitik nicht nur um komparative Kostenvorteile, sondern auch darum, wie die Gewinne verteilt werden. Wirtschaftskrieg um relative Gewinne ist real. Google, facebook, Amazon \& Co. hätten niemals diese Stellung in Europa erlangen können, wenn Europa mit derselben Konsequenz seine Datenschutzgesetze durchsetzen würde, wie die USA das für ihre Gesetze in anderen Bereichen gemacht haben. Das wäre nicht nur aus datenschutzrechtlichen Gründen dringend notwendig gewesen: Wenn sich einmal ein Oligopol wie im Falle der großen U.S.-Technologiekonzerne etabliert hat, ist es schwer zu knacken. Und der Mehrwert auch der heimischen Produktion wird abgezogen, in diesem Fall z. B. von den europäischen Telekom-Konzernen. So haben im Wesentlichen nur Russland (Yandex) und China (Baidu, Tencent) eigene Internetgiganten, obwohl Europa über einen Binnenmarkt verfügt, der größer ist als der chinesische.

In Deutschland propagierte Friedrich List die Idee von Schutzzöllen zur Entwicklung einer eigenen Industrie und legte diese Ideen ausführlich in seinem Hauptwerk „Das nationale System der politischen Ökonomie“ (List 1930) dar. Wenn das „System der Produktivkräfte" hinreichend entwickelt sei, könne man sich zunehmend dem Wettbewerb stellen. Zuvor hatte in den USA bereits 1791 Alexander Hamilton in seinem Bericht an den amerikanischen Kongress ähnliche Ideen vertreten. Ein Land müsse einen Gemeinwohlvertrag zum Wohle seiner Bürger abschließen. Auch diesen Vorschlägen lag die Vorstellung zugrunde, dass internationaler Handel auf Basis der Parität der Akteure unter Regeln zu erfolgen habe, die einen fairen Interessenausgleich bewirken.

\section{Ein Kompass für die deutsche Industrie und Politik in einer Ära steigender Unsicherheit}

Deutschland ist wie keine andere große Wirtschaftsnation mit der Weltwirtschaft verflochten. Alle Strukturveränderungen im Weltwirtschaftssystem werden uns zuerst stark (be)treffen. Der Brexit ist nur ein Symptom der Krise der globalen Handelsordnung. Die Grenzausgleichssteuer (Border Adjustment Tax, BAT), die zum Beispiel in den USA im Gespräch ist, wäre eine Maßnahme, die deutsche Wirtschaft weitaus stärker treffen würde (Tully 2017). Mit Exporten nach Großbritannien von 89 Mrd. $€$ und Importen von 38 Mrd. $€$ im Jahr 2015 war Großbritannien der drittgrößte Handelspartner Deutschlands. Der Handel mit den USA liegt um $40 \%$ höher.

Viele Produkte der deutschen Industrie haben Alleinstellungsmerkmale und sind relativ wenig preissensitiv. Auch nach einem erfolgten Brexit sehe ich hier keine allzu große Beeinträchtigung des deutschen Außenhandels, zumal Großbritannien 
viele Industrien einfach weitgehend fehlen. Selbst im Szenario des „harten Brexit“ würde Großbritannien versuchen, einen liberalen Handelsvertrag mit der EU zu schließen und die offenen Gütermärkte zu bewahren. ${ }^{2}$

Es ist dennoch richtig, dass sich Deutschland und die Europäische Union grundsätzliche Gedanken zu ihrer Strategie in einer sich verändernden Welt machen sollten. Dazu müssen europäische und deutsche Position entwickelt werden.

1. Bilateralismus und gesamteuropäische Politik: Mit dem Brexit und „America first" hat der Bilateralismus einen Schub bekommen. Bilateralismus muss nicht schlecht sein, aber er muss in geordneten Regeln ablaufen. Nationale Akteure sind allerdings schneller und wendiger als supranationale. Die Europäische Union und die Mitgliedstaaten werden darüber klar werden müssen, ob sie weiter gemeinsam als EU in diese Verhandlungen gehen wollen. Wenn das der Fall ist, müssen die Kompetenzen der entsprechenden Organe deutlich ausgeweitet werden.

2. Waffenparität bei Handelspolitik und Standards: Während die USA durch globale Anwendung nationalen Rechts Fakten schaffen, hat die Europäische Union dem nicht viel entgegenzusetzen. Das Ziel kann auch für die Europäische Union nur sein, den Marktzugang als Pfand für faire Lösungen einzusetzen. Hierzu gehört auch die Schaffung eines begünstigten Heimatmarktes für Technologiekonzerne. Eine Maßnahme wie die BAT (Border Adjustment Tax), die in den USA für Waren diskutiert wird, kann genauso gut bei Dienstleistungen erhoben werden. Bei der Border Adjustment Tax würde nicht der mit Importen erzielte Gewinn besteuert, sondern der Umsatz. Genauso gäbe es Steuervorteile für Exporte. Bei Waren wäre dies nachteilig für Europa bzw. Deutschland, bei Dienstleistungen für die USA.

3. Die wichtigeren Debatten mit Großbritannien werden sich um die Freizügigkeit von Personen und Dienstleistungsverkehr drehen, insbesondere um Finanzdienstleistungen. Es kann nicht übersehen werden, das Deutschland als die größte Wirtschaftsnation Europas mit dem Finanzplatz Frankfurt zunehmend als Finanzplatz auf einen nachrangingen Platz verwiesen wurde. In Europa ist London Finanzplatz Nummer eins. Dies ist umso paradoxer, als Großbritannien bis heute nicht Mitglied der Eurozone ist. Der Brexit gibt Deutschland nun die Chance, den Finanzplatz wieder zu stärken. Ein erstes Opfer des Brexit ist wahrscheinlich die Fusion der London Stock Exchange mit der Frankfurter Börse. Die Eigentümer haben ihre Zustimmung zu dieser Fusion zurückgezogen, seitdem klar ist, dass nach einem Brexit nicht London alleine Standort der Muttergesellschaft sein kann. Die hessische Landesregierung sowie der Verfasser sehen darin eine Chance, den Finanzplatz Frankfurt zu bewahren (BILD 2017). Der Brexit bietet damit die Chance, die Finanzmarktordnung zu überdenken und für Deutschland und Kontinentaleuropa angemessen zu gestalten. Dabei gilt es auch, zu berücksichtigen, dass Kontinentaleuropa ein eher kredit- als kapitalmarktbasiertes Finanzsystem hat (Otte 2010).

4. Kerneuropa: Aktuell wird die Idee eines „Kerneuropa“, eines „Europa der verschiedenen Geschwindigkeiten“ von Jean-Claude Juncker und Angela Merkel

\footnotetext{
2 Das ,hart“ bezieht sich vor allem auf die Freizügigkeit von Personen (Marquardt 2017).
} 
wiederbelebt (vks 2017). Nach dieser Idee sollen einige Staaten bei der Integration vorangehen können und andere folgten später. Erstmalig wurde sie von Karl Lamers und Wolfgang Schäuble 1994 vertreten, von Helmut Kohl damals aber vehement abgelehnt (Otte und Grewe 2000). Nun scheint die Zeit reif für eine Renaissance, und für den Verfasser überwiegen hier die Chancen. Zwar sind auch hier die Schwierigkeiten enorm, aber eine Kerngruppe von EU-Ländern hätte zumindest die Chance, in einigen Bereichen voranzuschreiten.

$\mathrm{Zu}$ diesen Punkten sollten sowohl die Bundesrepublik Deutschland als auch die Europäische Union unverzüglich eine Strategie entwickeln. Sonst setzt sich die Marginalisierung Europas fort.

Open Access Dieser Artikel wird unter der Creative Commons Namensnennung 4.0 International Lizenz (http://creativecommons.org/licenses/by/4.0/deed.de) veröffentlicht, welche die Nutzung, Vervielfältigung, Bearbeitung, Verbreitung und Wiedergabe in jeglichem Medium und Format erlaubt, sofern Sie den/die ursprünglichen Autor(en) und die Quelle ordnungsgemäß nennen, einen Link zur Creative Commons Lizenz beifügen und angeben, ob Änderungen vorgenommen wurden.

\section{Literatur}

Baudet, T. (2012). The significance of borders. Leiden: Brill.

BILD (2017). London und Frankfurt: Ist die Börsen-Fusion noch zu retten? http://www.bild.de/geld/ wirtschaft/brexit/ist-die-boersen-fusion-noch-zu-retten-50629934.bild.html (Erstellt: 27.02.).

Desjardins, J. (2015). China vs. United States - a tale of two economies. http://www.visualcapitalist.com/ china-vs-united-states-a-tale-of-two-economies/ (Erstellt: 15.10.).

Freytag, A., Draper, P., \& Fricke, S. (2014). Die Auswirkungen von TTIP. http://www.kas.de/wf/doc/kas_ 15293-1442-1-30.pdf?150311105611

Fuentes-Niva, R. et al. (2014). Working for the few - political capture and economic inequality. https:// www.oxfam.org/en/research/working-few (Erstellt: 20.01.).

Fukuyama, F. (1992). The end of history and the last man. New York: Free Press.

Gilpin, R. (1983). War and change in world politics. Cambridge: Cambridge University Press.

Gilpin, R. (1987). The political economy of international relations. Princeton: Princeton University Press.

Hulverscheidt, C. (2016). Populisten würden die Welt in Rezession und Arbeitslosigkeit stürzen. http:// www.sueddeutsche.de/wirtschaft/weltwirtschaft-populisten-wuerden-die-welt-in-rezession-undarbeitslosigkeit-stuerzen-1.3189565 (Erstellt: 05.10.).

Ivory, D., \& Vlasic, B. (2015). \$900 million penalty for G.M.'s deadly defect leaves many cold. https:// www.nytimes.com/2015/09/18/business/gm-to-pay-us-900-million-over-ignition-switch-flaw.html (Erstellt: 17.09.).

Jahberg, H. (2015). VW-Aktie: kaufen oder nicht. http://www.tagesspiegel.de/wirtschaft/volkswagen-vwaktie-kaufen-oder-nicht/12359884.html (Erstellt: 23.09.).

Maynard Keynes, J. (1933). National Self-Sufficiency. The Yale Review, 22(4), 755-769.

Kindleberger, C. (2013). The World in Depression (1. Aufl. 1973). Berkeley: University of California Press.

Korkery, M. (2016). Wells Fargo fined \$185 million for fraudulently opening accounts. https://www. nytimes.com/2016/09/09/business/dealbook/wells-fargo-fined-for-years-of-harm-to-customers. html?_r=0 (Erstellt: 08.09.).

List, F. (1930). Das nationale System der politischen Ökonomie. Berlin: v. Artur Sommer.

Marquardt, J.-P. (2017). Fahrplan zum Brexit liegt vor. https://www.tagesschau.de/ausland/brexit-whitepaper-101.html

von Marschall, C. (2016). So nah sind sich links und rechts. http://www.tagesspiegel.de/politik/nach-demparteitag-der-linken-so-nah-sind-sich-links-und-rechts/13660188.html (Erstellt: 30.05.).

Otte, M. (1998). The Euro and the future of the European Union. New York: American Council on Germany. Occassional Paper \#5, April

Otte, M. (2010). Finanzplatz Deutschland vs. deutsches Bankensystem - Zwei politökonomische Perspektiven für die Zukunft. In F. Keuper \& D. Puchta (Hrsg.), Deutschland 20 Jahre nach dem Mauerfall - Rückblick und Ausblick (S. 179-204). Wiesbaden: Springer. 
Otte, M. (2012). Globalisierung, mitteleuropäisches Wirtschaftsmodell und angelsächsischer Kapitalismus. Zur ökonomischen Selbstbehauptung Europas. In J. Pucher \& J. Frank (Hrsg.), Strategie und Sicherheit - Der Gestaltungsspielraum der österreichischen Sicherheitspolitik (S. 217-231). Wien: Böhlau.

Otte, M., \& Grewe, J. (2000). A rising middle power? German foreing policy in transformation 1989-1990. New York: St. Martin's Press.

Patton, M. (2016). U.S. role in global economy declines nearly 50. https://www.forbes.com/sites/ mikepatton/2016/02/29/u-s-role-in-global-economy-declines-nearly-50/\#77f517155e9e (Erstellt: 29.02.).

Ricardo, D. (1815). Essay on the influence of a low price of corn on the profits of stock

Ricardo, D. (1817). On the principles of political economy and taxation. London: John Murray.

Scheuer, T., Pfeifer, J., \& Kastrun, G. (2012). Permanente Krisen gibt es nicht - Wirtschaftspolitisches Forum des Graz Schumpeter Centre. https://static.uni-graz.at/fileadmin/presse/Archiv/0312/newswww_ archiv_detail_009.html (Erstellt: 27.03.).

Through, P.L. (2012). Securities Exchange Act Of 1934. https://www.sec.gov/about/laws/sea34.pdf

Sinn, H.-W. (2017). Krisentheorie - Marx’ wahre Leistung. http://www.zeit.de/2017/05/krisentheoriekarl-marx-finanzpolitik-europaeische-zentralbank (Erstellt: 13.02.).

Streek, W. (2016). How will capitalism end? Brooklyn: Verso Books.

Tully, S. (2017). The promise and the peril of the Trump economy. Fortune, 3, 31-38.

vks, dpa, Reuters, \& AFP (2017). Juncker will EU radikal reformieren. http://www.spiegel.de/politik/ ausland/jean-claude-juncker-wirbt-fuer-kerneuropa-radikale-eu-reform-a-1136086.html. Zugegriffen: 25.2.2017

Wagenknecht, S. (2016). Reichtum ohne Gier - wie wir uns vor dem Kapitalismus retten. Frankfurt: Campus.

Wikipedia (2017) https://en.wikipedia.org/w/index.php?title=Corn_Laws\&amp;oldid=768385094. Zugegriffen: 25.02 .2017 . 